\title{
Dispersion relations and subtractions in hard exclusive processes
}

\author{
I.V. Anikin, O.V. Teryaev \\ Bogoliubov Laboratory of Theoretical Physics, JINR, 141980 Dubna, Russia
}

\begin{abstract}
We study analytical properties of the hard exclusive process amplitudes. We found that QCD factorization for deeply virtual Compton scattering and hard exclusive vector meson production results in the subtracted dispersion relation with the subtraction constant determined by the Polyakov-Weiss $D$-term. The relation of this constant to the fixed pole contribution found by Brodsky, Close and Gunion and defined by parton distributions is studied and proved for momentum transfers exceeding the typical hadronic scale. The continuation to the real photons limit is considered and the numerical correspondence between lattice simulations of $D$-term and low energy Thomson amplitude is found. For sufficiently large $t$ the subtraction may be expressed in the form similar to suggested earlier for real Compton scattering.
\end{abstract}

\section{INTRODUCTION}

Hard exclusive reactions described by the Generalized Parton Distributions (GPDs) [1, 2, 3, 3, 4, 5, 6] are the subject of extensive theoretical and experimental studies. The analytical properties of deeply virtual Compton scattering (DVCS) and hard exclusive vector meson production (VMP) amplitudes [7, 8, 9, 10] constitute the important aspect of these studies. They play also the major role in the hadronic processes such as the nucleon-nucleon scattering at very high energies to be studied at LHC and in non-accelerator experiments [11].

The crucial point in application of the relevant dispersion relations is a possible ambiguity due to the subtraction constants which are the counterparts of the normalization constants implied by the ultraviolet renormalization procedure. An attractive possibility is represented by cases when such constants are defined by the imaginary part of the amplitudes. This situation was explored long ago in the case of forward Compton amplitude 12, 13, 14, and was recently reconsidered for DVCS 10].

In this paper we address the problem of dispersion relations and subtractions in the framework of the leading order QCD factorization. We find that it leads to subtracted dispersion relations with the subtraction constant defined by the Polyakov-Weiss $D$ term [15]. At the same time, for $t$ exceeding typical hadronic scale we relate the subtraction constant to the integrals of parton distribution at zero skewness.

\section{DISPERSION RELATION IN THE SKEWNESS PLANE}

We restrict our study by the case of large $s$ and $Q^{2}$ and small $t \ll s, Q^{2}$, where QCD factorization is applicable. At the leading order, this results in the following expressions for DVCS and vector $\left(\rho^{0}\right)$ meson production amplitudes:

$$
T_{D V C S}^{\mu \nu}=\frac{g_{\perp}^{\mu \nu}}{2} \bar{u}\left(p_{2}\right) \hat{n} u\left(p_{1}\right) \sum_{f=u, d, s, \ldots} e_{f}^{2} \mathcal{A}_{f}(\xi, t)
$$

and

$$
\begin{aligned}
& T_{V M P}^{\mu}=\frac{\alpha_{s} f_{\rho} C_{F} e_{L}^{\mu}}{\sqrt{2} N_{c} Q} \bar{u}\left(p_{2}\right) \hat{n} u\left(p_{1}\right) \\
& \times \mathcal{V}\left[e_{u} \mathcal{A}_{u}(\xi, t)-e_{d} \mathcal{A}_{d}(\xi, t)\right]
\end{aligned}
$$

with the GPDs part (which may be interpreted as a weighted handbag diagram, i.e. the coupling of local quark currents to two photons)

$$
\mathcal{A}_{f}(\xi, t)=\int_{-1}^{1} d x \frac{H_{f}^{(+)}(x, \xi, t)}{x-\xi+i \epsilon}
$$

and the meson part for VMP case

$$
\mathcal{V}=\int_{0}^{1} d y \frac{\phi_{1}(y)}{y} .
$$

In (3), $H^{(+)}(x, \xi, t)$ denotes the singlet $(C=+1)$ combination of GPDs, summing the contributions of quarks and anti-quarks and of $s$ - and $u$-channels:

$$
H_{f}^{(+)}(x, \xi, t)=H_{f}(x, \xi, t)-H_{f}(-x, \xi, t) .
$$

For the sake of brevity, we will keep only the dependence of GPDs on the skewness $\xi=Q^{2} /\left(2 s+Q^{2}\right)$. For $\xi \rightarrow 0$, (3) may acquire divergencies at $x=0$, which will be one of objects of our analysis.

Contrary to the forward case, expression (3) does not have a form of the dispersion relation because of the appearance of $\xi$ in the numerator. Nevertheless, the amplitude (3) as a function of $\xi$ manifests the analyticity in the unphysical region $|\xi|>1$ [9]. This 
region is associated with the contribution of Generalized Distribution Amplitudes (GDAs) [16] related to GPDs by crossing [17]. To prove the analyticity of the amplitude for $|\xi|>1$, one represents the denominator of (3) as the geometric series:

$$
\mathcal{A}(\xi)=-\sum_{n=0}^{\infty} \xi^{-n-1} \int_{-1}^{1} d x H^{(+)}(x, \xi) x^{n} .
$$

This series is convergent 9 thanks to the polynomiality condition (see e.g. [5, 6$]$ ):

$$
\int_{-1}^{1} d x x^{n} H(x, \xi)=\sum_{k=0,2 \ldots}^{n} \xi^{k} A_{k}+\frac{1-(-1)^{n}}{2} \xi^{n+1} C .
$$

One may now easily calculate the discontinuity across the cut $-1<\xi<1$ and write the fixed- $t$ dispersion relation [9] for the leading order amplitude (3) in the skewness plane:

$$
\operatorname{Re} \mathcal{A}(\xi)=\frac{\mathcal{P}}{2 \pi i} \int_{-1}^{1} d x \frac{\operatorname{Disc} \mathcal{A}(x)}{x-\xi}+\Delta(\xi) .
$$

or, using (3),

$$
\mathcal{P} \int_{-1}^{1} d x \frac{H^{(+)}(x, \xi)}{x-\xi}=\mathcal{P} \int_{-1}^{1} d x \frac{H^{(+)}(x, x)}{x-\xi}+\Delta(\xi),
$$

where $\Delta(\xi)$ is a possible subtraction. This expression represents the holographic property of GPD: the relevant information about hard exclusive amplitudes in the considered leading approximation is contained in the one-dimensional sections $x= \pm \xi$ of the two dimensional space of $x$ and $\xi$. These holographic as well as tomographic 17] properties in momentum space are complementary to the often discussed holography and tomography in coordinate space [6].

We are now going to prove that $\Delta(\xi)$ is finite and independent of $\xi$, i.e. $\Delta(\xi)=$ const. To do this, one considers the following representation:

$$
\begin{gathered}
\Delta(\xi)=\mathcal{P} \int_{-1}^{1} d x \frac{H^{(+)}(x, \xi)-H^{(+)}(x, x)}{x-\xi}= \\
-\left.\mathcal{P} \int_{-1}^{1} d x \sum_{n=1}^{\infty} \frac{1}{n !} \frac{\partial^{n}}{\partial \xi^{n}} H^{(+)}(x, \xi)\right|_{\xi=x}(\xi-x)^{n-1} .
\end{gathered}
$$

Due to the polynomiality condition the only surviving highest power term in this series is equal to a finite subtraction constant. This can also be derived with a use of the Double Distributions (DDs) formalism. Namely, the $H^{(+)}(x, \xi)$ is expressed through the corresponding DDs as

$$
\begin{aligned}
& H^{(+)}(x, \xi)=\int_{-1}^{1} d \alpha \int_{-1+|\alpha|}^{1-|\alpha|} d \beta[f(\alpha, \beta)+\xi g(\alpha, \beta)] \\
& \times[\delta(x-\alpha-\xi \beta)-\delta(-x-\alpha-\xi \beta)] .
\end{aligned}
$$

Substituting this expression into (9), one gets that the $f(\alpha, \beta)$-terms which depend on $\xi$ are cancelled and eqn. (9) becomes $\xi$-independent (cf. [9]):

$$
\Delta(\xi)=-2 \int_{-1}^{1} d \alpha \int_{-1+|\alpha|}^{1-|\alpha|} d \beta \frac{g(\alpha, \beta)}{1-\beta} \equiv \Delta .
$$

Notice that the cancellation of $f(\alpha, \beta)$ and validity of (11) are not spoiled (provided $\xi \neq 0$ ), even if the singularity corresponding to $f(\alpha, \beta) \sim \alpha^{-a}$ is present.

In (11), one can choose

$$
g(\alpha, \beta)=\delta(\alpha) D(\beta),
$$

where the function $D(\beta)$ is $D$-term 15 . The assumption (12) is a result of the corresponding "gauge" [17 as discussed also in [6]. With (12), the $\Delta$ term takes the following form:

$$
\Delta=2 \int_{-1}^{1} d \beta \frac{D(\beta)}{\beta-1}
$$

It should be emphasized that both integrals in (8) are divergent at $\xi=t=0$, and this divergencies do not cancel for $\xi \rightarrow 0$. It means that $\Delta$ is not defined for $\xi=t=0$ [26]. On the other hand, for an arbitrarily small $\xi$ the integrals in (8) are finite and, therefore, $\Delta$ is well-defined. Note some similarity between (13) and (3) so that $\Delta$ may be also interpreted as a contribution of local two-photon coupling to the quark currents which is independent of $\xi$.

Taking into account the parameterization [15]

$$
D(\beta)=\left(1-\beta^{2}\right) \sum_{n=0}^{\infty} d_{n} C_{2 n+1}^{(3 / 2)}(\beta),
$$

and keeping only the lowest term, one gets

$$
\Delta=-4 d_{0} .
$$

This lowest term $d_{0}$ was estimated within the framework of different models. We focus on the results of 
chiral quark-soliton model [18]: $d_{0}^{\mathrm{CQM}}\left(N_{f}\right)=d_{0}^{u}=$ $d_{0}^{d}=-\frac{4.0}{N_{f}}$, where $N_{f}$ is the number of active flavours, and lattice simulations [19]: $d_{0}^{\text {latt }}=d_{0}^{u} \approx d_{0}^{d}=-0.5$. The subtraction constant varies as

$$
\begin{aligned}
& \Delta_{\mathrm{CQM}}^{p}(2)=\Delta_{\mathrm{CQM}}^{n}(2) \approx 4.4, \\
& \Delta_{\text {latt }}^{p} \approx \Delta_{\text {latt }}^{n} \approx 1.1
\end{aligned}
$$

for the DVCS on both the proton and neutron targets.

\section{DISPERSION RELATION IN THE $\nu$ PLANE}

We have seen that the $D$-term determines the finite subtraction in the dispersion relation in the skewness plane. Let us now compare the dispersion relation (8) with the dispersion relation written in the $\nu$ plane 8 where $\nu=(s-u) / 4 m_{N}$. In terms of the new variables $\nu^{\prime}, \nu$ related to $x, \xi$ as

$$
x^{-1}=\frac{4 m_{N} \nu^{\prime}}{Q^{2}}, \quad \xi^{-1}=\frac{4 m_{N} \nu}{Q^{2}},
$$

the fixed- $t$ dispersion relation becomes the subtracted one:

$$
\begin{aligned}
& \operatorname{Re} \mathcal{A}\left(\nu, Q^{2}\right)=\frac{\nu^{2}}{\pi} \mathcal{P} \int_{\nu_{0}}^{\infty} \frac{d \nu^{\prime 2}}{\nu^{\prime 2}} \frac{\operatorname{Im} \mathcal{A}\left(\nu^{\prime}, Q^{2}\right)}{\left(\nu^{\prime 2}-\nu^{2}\right)}+\Delta= \\
& \frac{\mathcal{P}}{\pi} \int_{\nu_{0}}^{\infty} d \nu^{\prime 2} \operatorname{Im} \mathcal{A}\left(\nu^{\prime}, Q^{2}\right)\left[\frac{1}{\nu^{\prime 2}-\nu^{2}}-\frac{1}{\nu^{\prime 2}}\right]+\Delta .(18)
\end{aligned}
$$

Here, $\nu_{0}=Q^{2} / 4 m_{N}$ (the $Q^{2}$ dependence here is shown explicitly) and the nucleon pole term residing in this point may be considered separately [8].

This subtracted (in the symmetric unphysical point $\nu=0$ ) dispersion relation is the principal result of our paper. It is applicable for both DVCS (cf. 7, 8] ) and VMP (cf. [20]) amplitudes.

It can be considerably simplified provided $\operatorname{Im} \mathcal{A}(\nu)$ decreases fast enough so that both terms in the squared brackets can be integrated separately:

$$
\operatorname{Re} \mathcal{A}(\nu)=\frac{\mathcal{P}}{\pi} \int_{\nu_{0}}^{\infty} d \nu^{\prime 2} \frac{\operatorname{Im} \mathcal{A}\left(\nu^{\prime}\right)}{\nu^{\prime 2}-\nu^{2}}+\mathbf{C}_{0}
$$

where

$$
\begin{aligned}
\mathbf{C}_{0} & =\Delta-\frac{\mathcal{P}}{\pi} \int_{\nu_{0}}^{\infty} d \nu^{\prime 2} \frac{\operatorname{Im} \mathcal{A}\left(\nu^{\prime}\right)}{\nu^{\prime 2}} \\
& =\Delta+\mathcal{P} \int_{-1}^{1} d x \frac{H^{(+)}(x, x)}{x}
\end{aligned}
$$

Now, using (9) with $\xi=0$, one gets:

$$
\begin{aligned}
\Delta & =\mathcal{P} \int_{-1}^{1} d x \frac{H^{(+)}(x, 0)-H^{(+)}(x, x)}{x} \\
& =2 \mathcal{P} \int_{-1}^{1} d x \frac{H(x, 0)-H(x, x)}{x},
\end{aligned}
$$

where the symmetry property arising from the $T$ invariance: $H(x,-x)=H(x, x)$ is used. The relation (21) can also be obtained from the "sum rules" [4]:

$$
\int_{-1}^{1} d x \frac{H(x, \xi+x z)-H(x, \xi)}{x}=\sum_{n=1}^{\infty} z^{n} \int_{-1}^{1} d x x^{n-1} D(x)
$$

for $\xi=0$ and $z=1$.

Let us stress that for the valence $(C=-1)$ contributions to the amplitudes of the hard exclusive production of, say, pions [4] and exotic hybrid mesons 21], $\Delta=0$ because of the mentioned symmetry in $x$ and the $\xi$-independence.

Substituting (21) into (20), one can see that the $D$-term is cancelled from the expression for the subtraction constant

$$
\mathbf{C}_{0}(t)=2 \mathcal{P} \int_{-1}^{1} d x \frac{H(x, 0, t)}{x},
$$

where we restored the dependence on $t$ which was omitted for brevity. This constant is similar to the result obtained in the studies of the fixed pole contribution to the forward Compton amplitude [12]. At the same time, at $\xi, t=0$, GPDs are expressed in terms of standard parton distributions $H(x, 0)=$ $q(x) \theta(x)-\bar{q}(-x) \theta(-x)$. Formally one has

$$
\begin{aligned}
\mathbf{C}_{0}(0) & =2 \int_{0}^{1} d x \frac{q(x)+\bar{q}(x)}{x} \\
& =2 \int_{0}^{1} d x \frac{q_{v}(x)+2 \bar{q}(x)}{x} .
\end{aligned}
$$

However, the integral defining $\mathbf{C}_{0}(0)$ diverges at low $x$ in both the valence and sea quark contributions. Therefore, for $t=0$ we should consider (18) as a correct general form of the dispersion relation which includes the infinite subtraction at the point $\nu=0$ and the subtraction constant associated with the $D$-term.

For $t \neq 0$, the integral in (23) converges for sufficiently large $t$. In the case of Regge inspired parameterization [4] $H(x, 0,-t) \sim x^{-\alpha(0)+\alpha^{\prime} t}$, this condition 
reads as $t>\alpha(0) / \alpha^{\prime}$, resulting in $t \gtrsim 1(10) \mathrm{GeV}^{2}$ for the valence (sea) quark distributions.

The divergence of (23) was originally discussed within the framework of the parton model and its modifications 12] while we address this problem in the framework of the leading order QCD factorization. Therefore, our result (18), although being formally $\xi$ independent, cannot, generally speaking, be continued to the forward limit $\xi \sim Q^{2} \rightarrow 0$ (s=const). The limit $\xi \rightarrow 0, s \rightarrow \infty, Q^{2}=$ const still corresponds to the highly non-forward kinematics with the masses of initial and final photons being rather different. At the same time, further exploration of the possible manifestation of the $D$-term in the forward Compton scattering seems very interesting.

The formal continuation of (18) would result in the following subtracted dispersion relation for the forward Compton scattering amplitude:

$$
\operatorname{Re} \mathcal{A}(\nu)=\frac{\nu^{2}}{\pi} \mathcal{P} \int_{0}^{\infty} \frac{d \nu^{\prime 2}}{\nu^{\prime 2}} \frac{\operatorname{Im} \mathcal{A}\left(\nu^{\prime}\right)}{\left(\nu^{\prime 2}-\nu^{2}\right)}+\Delta
$$

Comparing this expression with the dispersion relation for the forward Compton scattering amplitude 22], one can observe an interesting numerical coincidence. For the proton target, our subtraction combined with the lattice simulations (16) is rather close to the low energy Thomson term (note that $\Delta_{\text {Thomson }}=1$ for our normalization of the Compton amplitude). As a result, the mysterious occurrence of Thomson term at large energies 22] can now be supplemented with its possible appearance also at large $Q^{2}$. This does not hold in the case of neutron target where DVCS subtraction term is the same, while Thomson term is zero. This may be because the sum of squares of valence quark charges is equal to the square of proton charge, i.e. to the square of their sum (cf. 23] ), whereas for the neutron these quantities differ.

\section{CONCLUSIONS}

In this paper we show that the fixed- $t$ dispersion relations for the DVCS and VMP amplitudes require the infinite subtractions at the unphysical point $\nu=0$ with the subtraction constants associated with the $D$ terms. However, for the productions of the mesons defined by valence $(C=-1)$ GPDs the finite subtraction is absent.

We also show that the appearance of the subtraction expressed in terms of (forward) parton distributions [12] may be investigated in the framework of the leading order QCD factorization. We consider the possibility of continuation of our results to the real photons limit. The surprising similarity between the lattice simulations of the $D$-term and the low energy Thomson amplitude in the proton target case is found.

\section{ACKNOWLEDGMENTS}

We would like to thank A.P. Bakulev, M. Diehl, A.V. Efremov, S.B. Gerasimov, D.Yu. Ivanov, D. Müller, B. Pire, M.V. Polyakov, A.V. Radyushkin, L. Szymanowski, S. Wallon for useful discussions and correspondence. This work was supported in part by Deutsche Forschungsgemeinschaft (Grant 436 RUS 113/881/0), RFBR (Grants 06-02-16215 and 07-0291557) and RF MSE RNP (Grant 2.2.2.2.6546).

Note added. - After this work was completed, the papers [24, 25] appeared confirming our results and generalizing them to the next-to-leading order.

[1] D. Mueller, D. Robaschik, B. Geyer, F. M. Dittes and J. Horejsi, Fortsch. Phys. 42, 101 (1994) arXiv:hep-ph/9812448.

[2] X. D. Ji, Phys. Rev. D 55, 7114 (1997) arXiv:hep-ph/9609381.

[3] A. V. Radyushkin, Phys. Rev. D 56, 5524 (1997) arXiv:hep-ph/9704207.

[4] K. Goeke, M. V. Polyakov and M. Vanderhaeghen, Prog. Part. Nucl. Phys. 47, 401 (2001) arXiv:hep-ph/0106012.

[5] M. Diehl, Phys. Rept. 388, 41 (2003) arXiv:hep-ph/0307382.

[6] A. V. Belitsky and A. V. Radyushkin, Phys. Rept. 418, 1 (2005) arXiv:hep-ph/0504030.

[7] B. Pasquini, M. Gorchtein, D. Drechsel, A. Metz and M. Vanderhaeghen, Eur. Phys. J. A 11, 185 (2001) arXiv:hep-ph/0102335.

[8] D. Drechsel, B. Pasquini and M. Vanderhaeghen, Phys. Rept. 378, 99 (2003) arXiv:hep-ph/0212124.

[9] O. V. Teryaev, "Analytic properties of hard exclusive amplitudes," in proc. of 11th Intern. Conf. on Elastic and Diffractive Scattering (Chateau de Blois, May 2005), ed. by M. Haguenauer, B.Nicolescu, J. Tr̂an Thanh Vân, p. 237 arXiv:hep-ph/0510031.

[10] K. Kumericki, D. Muller and K. Passek-Kumericki, arXiv:hep-ph/0703179

[11] M. M. Block, Phys. Rept. 436, 71 (2006) arXiv:hep-ph/0606215.

[12] S. J. Brodsky, F. E. Close and J. F. Gunion, Phys. Rev. D 5, 1384 (1972); ibid. D 8, 3678 (1973).

[13] S. Y. Lee, Nucl. Phys. B 45, 449 (1972).

[14] S. B. Gerasimov, arXiv:hep-ph/0701073

[15] M. V. Polyakov and C. Weiss, Phys. Rev. D 60, 114017 (1999) arXiv:hep-ph/9902451. 
[16] M. Diehl, T. Gousset, B. Pire and O. Teryaev, Phys. Rev. Lett. 81, 1782 (1998) arXiv:hep-ph/9805380.

[17] O. V. Teryaev, Phys. Lett. B 510, 125 (2001) arXiv:hep-ph/0102303.

[18] V. Y. Petrov, P. V. Pobylitsa, M. V. Polyakov, I. Bornig, K. Goeke and C. Weiss, Phys. Rev. D 57, 4325 (1998) arXiv:hep-ph/9710270.

[19] M. Gockeler, R. Horsley, D. Pleiter, P. E. L. Rakow, A. Schafer, G. Schierholz and W. Schroers [QCDSF Collaboration], Phys. Rev. Lett. 92, 042002 (2004) arXiv:hep-ph/0304249.

[20] S. J. Brodsky and F. J. Llanes-Estrada, Eur. Phys. J. C 46, 751 (2006) arXiv:hep-ph/0512247; M. Vanderhaeghen, P. A. M. Guichon and M. Guidal, Phys. Rev. D 60, 094017 (1999) arXiv:hep-ph/9905372.
[21] I. V. Anikin, B. Pire, L. Szymanowski, O. V. Teryaev and S. Wallon, Phys. Rev. D 70, 011501 (2004) arXiv:hep-ph/0401130; Phys. Rev. D 71, 034021 (2005) arXiv:hep-ph/0411407; Nucl. Phys. A 755, 561 (2005) arXiv:hep-ph/0501119]; arXiv:hep-ph/0509245

[22] M. Damashek and F. J. Gilman, Phys. Rev. D 1, 1319 (1970).

[23] F. E. Close and N. Isgur, Phys. Lett. B 509, 81 (2001) arXiv:hep-ph/0102067.

[24] M. Diehl and D. Y. Ivanov, arXiv:0707.0351 [hep-ph].

[25] M. V. Polyakov, arXiv:0707.2509 [hep-ph].

[26] Note that the point $\xi=0$ (or $Q^{2}=0$ ) cannot be accessed in DVCS and VMP experiments 